\title{
AFASTAMENTOS DO TRABALHO NA ENFERMAGEM: OCORRÊNCIAS COM TRABALHADORES DE UM HOSPITAL DE ENSINO'1
}

Denise Beretta Barboza ${ }^{2}$

Zaida Aurora Sperli Geraldes Soler ${ }^{3}$

Barboza DB, Soler ZASG. Afastamentos do trabalho na enfermagem: ocorrências com trabalhadores de um hospital de ensino. Rev Latino-am Enfermagem 2003 março-abril; 11(2):177-83.

As condições laborais da equipe de enfermagem freqüentemente são marcadas por riscos, os quais repercutem em elevado absenteísmo e licenças-saúde. O objetivo deste estudo foi caracterizar os afastamentos entre trabalhadores de enfermagem de um hospital geral de ensino da cidade de São José do Rio Preto-SP, registrados durante o ano de 1999. Tratase de uma investigação epidemiológica censitária, cuja coleta de dados foi efetuada por meio de consulta aos registros do Centro de Atendimento ao Trabalhador do hospital estudado. Os resultados indicaram que, no período, 333 trabalhadores envolveram-se em 662 episódios de afastamento. As licenças-saúde ocorreram, principalmente, por problemas geniturinário e doenças mal definidas entre enfermeiros, técnicos e auxiliares de enfermagem; e doenças dos órgãos dos sentidos, doenças infecta-parasitárias e doenças respiratórias entre os atendentes de enfermagem. Os dados obtidos subsidiam melhor análise dessa situação neste hospital e intervenções para melhoria das condições de trabalho vigentes.

DESCRITORES: absenteísmo, equipe de enfermagem, licença por motivo de saúde

\section{NURSING ABSENTEEISM: OCCURRENCES AT A UNIVERSITY HOSPITAL}

The nursing work conditions are surrounded by physical and chemical risks that result in a high number of absences as well as leaves. The purpose of this study was to characterize the absences of nursing workers from a University Hospital located in the city of São José do Rio Preto-SP during the year of 1999. This is a census epidemiological investigation. Data were collected from registries of the Hospital Workers' Attendance Center. Results showed that 333 workers were involved in 662 episodes of absences during this period. Sick leaves of nurses, nursing technicians and auxiliaries occurred mainly due to genitourinary tract disorders and other diseases. With respect to nursing aids, the causes of absences were: disturbances in sensory organs, infectious parasitic and respiratory diseases. These data enabled a better analysis of that hospital's environment as well as the improvement of these professionals' work conditions.

DESCRIPTORS: absence, nursing teams, leave

\section{RETIROS DEL TRABAJO EN LA ENFERMERÍA: OCURRENCIAS EN TRABAJADORES EN UN HOSPITAL DE ENSEÑANZA}

Las condiciones laborales del equipo de enfermería son frecuentemente marcadas por riesgos, situación que se refleja en un alto ausentismo e incapacidades médicas. El objetivo de este estudio fué caracterizar los retiros entre los trabajadores del equipo de enfermería de un hospital general de enseñanza de la ciudad de São José do Rio Preto-SP registrados durante el año de 1999. Se trató de una investigación por censo epidemiológico, cuya recolección de datos fue efectuada a través de la consulta a los registros del Centro de Atención al Trabajador del hospital estudiado. Los resultados indicaron que en este período 333 trabajadores estuvieron involucrados en 662 episodios de retiros. Las incapacidades ocurrieron principalmente por problemas génitourinarios y enfermedades mal definidas entre enfermeros, técnicos y auxiliares de enfermería; enfermedades de los órganos de los sentidos, infecto parasitárias y respiratórias dentro los ayudantes de enfermería. Los datos obtenidos aclaran mejor el análisis de esta situación en este hospital y demuestran la necesidad de intervenciones para la mejoría de las condiciones de trabajo vigentes.

DESCRIPTORES: ausentismo, equipo de enfermería, incapacidad médica

\footnotetext{
${ }^{1}$ Parte da dissertação de mestrado em andamento, junto ao Programa de Pós-Graduação da Faculdade de Medicina de São José do Rio Preto/ SP, este trabalho foi premiado em $1^{\circ}$ lugar, na categoria Pôster, no VIII ECIF-FAMERP, outubro/2000; ${ }^{2}$ Enfermeira do Trabalho, Mestranda em Enfermagem, Docente do Curso de Graduação em Enfermagem, e-mail: deniseberetta@bol.com.br; ${ }^{3}$ Enfermeira do Trabalho, Doutor em Enfermagem, Docente e Coordenador Geral do Curso de Graduação em Enfermagem, Membro do Corpo Docente e de Orientadores da PósGraduação. Faculdade de Medicina de São José do Rio Preto
} 
INTRODUÇÃO

No contexto hospitalar, a enfermagem constituise na maior força de trabalho, e suas atividades são freqüentemente marcadas por divisão fragmentada de tarefas, rígida estrutura hierárquica para o cumprimento de rotinas, normas e regulamentos, dimensionamento qualitativo e quantitativo insuficiente de pessoal, situação de exercício profissional que tem repercutido em elevado absenteísmo e afastamentos por doenças.

Usualmente, a ausência ao trabalho é denominada absenteísmo, apesar de, por definição, absentismo significar "o hábito de não comparecer, de estar ausente". Então, neste estudo, os dois termos serão usados com significado similar ${ }^{(1)}$.

Vários autores destacam que as condições de trabalho vivenciadas por muitos trabalhadores da equipe de enfermagem, particularmente em ambiente hospitalar, têm Ihes ocasionado problemas de saúde, freqüentemente relacionados à situação e setor de trabalho, provocando prejuízos pessoais, sociais e econômicos ${ }^{(2-6)}$.

$\mathrm{Na}$ análise dos aspectos que podem influenciar as atividades no trabalho, abordam-se os fatores intra, peri e extralaborais como: ambiente físico; riscos ocupacionais; higiene, estruturação e segurança do setor de trabalho; situação social de vida e do processo de trabalho do funcionário; organização e divisão das atividades; os meios disponíveis para o exercício profissional; jornada, turno, alimentação e transporte; situação social de moradia e deslocamento; interação pessoal e as relações entre produção e salário ${ }^{(7-8)}$.

Tratando-se especificamente do ambiente hospitalar, muito se tem falado e publicado a respeito das condições inadequadas de trabalho vigentes em grande parte dessas instituições, expondo seus trabalhadores a riscos de ordem biológica, física, química, ergonômica, mecânica, psicológica e social. Os hospitais constituemse em locais de aglutinação de pacientes/clientes acometidos por diferentes problemas de saúde, assistidos por trabalhadores diversos, da área da saúde ou técnicoadministrativas, e vários estudos têm apontado que os serviços de saúde, em particular os hospitais, geralmente proporcionam aos seus trabalhadores, principalmente da enfermagem, piores condições de trabalho em relação a outros serviços $^{(9-14)}$.

Como conseqüência das condições inseguras de trabalho em instituições hospitalares, no caso da equipe de enfermagem, têm sido freqüentes os acidentes de trabalho, o absenteísmo e os afastamentos por doenças, o que tem dificultado a organização do trabalho em diversos setores e, conseqüentemente, a qualidade da assistência de enfermagem prestada.

O estudo das relações entre saúde-doençatrabalho tem merecido a atenção de muitos pesquisadores na área de saúde ocupacional, destacando-se como prejuízos à saúde física e mental dos trabalhadores: prolongadas jornadas de trabalho; ritmo acelerado de produção, por excesso de tarefas; automação por realização de ações repetitivas com parcelamento de tarefas e remuneração baixa, em relação à responsabilidade e complexidade das tarefas executadas. Em tais situações, muitas vezes o trabalho deixa de significar satisfação, ganhos materiais e serviços sociais úteis, para tornar-se sofrimento, exploração, doença e morte.

Freqüentemente, os trabalhadores de enfermagem estão sujeitos a condições inadequadas de trabalho, provocando agravos à saúde, que podem ser de natureza física ou psicológica, gerando transtornos alimentares, de sono, de eliminação, fadiga, agravos nos sistemas corporais, diminuição do estado de alerta, estresse, desorganização no meio familiar e neuroses, fatos que, muitas vezes, levam a acidentes de trabalho e licenças para tratamento de saúde. Alguns autores destacam os fatores ergonômicos relacionados a problemas ósteomúsculo-articulares entre trabalhadores de enfermagem ${ }^{(15-}$ 17). Os agentes psicossociais causadores de danos à saúde dos trabalhadores de enfermagem associam-se ao contato freqüente com o sofrimento e a morte ${ }^{(9)}$; a monotonia de atividades repetitivas e parceladas e turnos rotativos de trabalho ${ }^{(18)}$; fadiga que leva ao estresse ${ }^{(2)}$.

Ante o exposto, como enfermeiras do trabalho e diante de nossa experiência profissional em hospitais, em especial no hospital-campo deste estudo, a questão norteadora deste trabalho é a preocupação com a situação de trabalho e o processo do adoecer entre trabalhadores de enfermagem desse hospital. Nesse contexto, foi objetivo desta pesquisa:

- descrever os afastamentos do trabalho entre trabalhadores de enfermagem de um hospital geral de ensino da cidade de São José do Rio Preto, registrados no Centro de Atendimento ao Trabalhador (CEAT), no ano de 1999. 


\section{MÉTODO}

Trata-se de uma investigação epidemiológica censitária, por meio de análise retrospectiva do ano de 1999, sobre a situação de afastamentos entre trabalhadores de enfermagem de um hospital de ensino. Ressalta-se que o estudo epidemiológico é explicativo e também tem finalidade descritiva, mostrando questionamentos do fenômeno pesquisado quanto ao tempo, espaço, características, qual o ponto focal da questão e se existem fatos circunstanciais ${ }^{(19)}$.

Este estudo foi realizado em um hospital geral, de grande porte, da cidade de São José do Rio Preto, região noroeste do estado de São Paulo, que tem finalidades de prestação de assistência complexa e especializada, de ensino e de pesquisa. Em 1999, esse hospital contava com 700 trabalhadores de enfermagem, sendo 103 enfermeiros, 31 técnicos de enfermagem, 410 auxiliares de enfermagem e 156 atendentes de enfermagem, a maioria com turnos de trabalho de 36 horas semanais, nos seguintes horários: manhã (7:00 às 13:00 horas); tarde (13:00 às 19:00 horas) e noite (19:00 às 07:00 horas - dias alternados - 12/36 horas).

Foram analisados os afastamentos do trabalho de profissionais de enfermagem registrados no Centro de Atendimento ao Trabalhador (CEAT) desse hospital, revelando-se que 333 trabalhadores foram acometidos, sendo 33 enfermeiros, 4 técnicos de enfermagem, 274 auxiliares de enfermagem, 22 atendentes de enfermagem. É importante esclarecer que esses 333 funcionários envolveram-se, nesse ano, em 662 episódios de afastamentos, o que deixa claro que alguns tiveram mais de um afastamento.

Preservando-se os aspectos éticos de pesquisa envolvendo seres humanos, este projeto foi inicialmente encaminhado ao Comitê de Ética e Pesquisa (CEP), para apreciação e aprovação, com prévia anuência da chefia

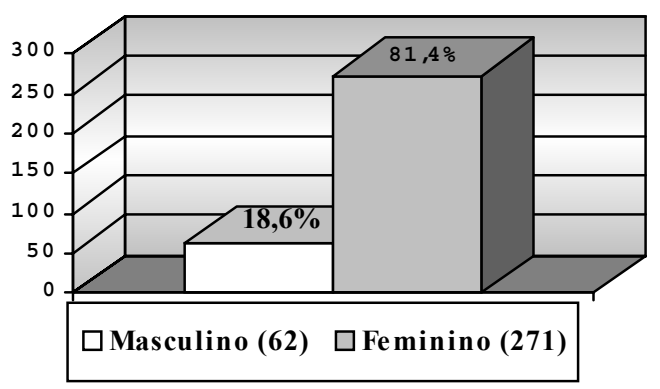

Figura 1 - Trabalhadores de enfermagem afastados do trabalho em 1999, segundo sexo do hospital. De posse da autorização do CEP, procedeuse à coleta dos dados junto ao CEAT da referida instituição, tendo por base um formulário elaborado segundo dados da ficha de atendimento individual do funcionário do ambulatório de saúde ocupacional. Os dados obtidos foram organizados e analisados no programa Excel/98, sendo configurados em forma de Tabelas e Figuras.

\section{RESULTADOS E DISCUSSÃO}

Os resultados obtidos foram agrupados segundo a caracterização da população e quanto aos diferentes afastamentos do trabalho ocorridos em 1999, entre os trabalhadores de enfermagem.

Caracterização da população: As Figuras 1 e 2 e a Tabela 1 mostram as características dos sujeitos deste estudo. Nota-se a predominância de trabalhadores de enfermagem do sexo feminino, pois, dos 333 funcionários da enfermagem afastados do trabalho, $271(81,4 \%)$ foram mulheres. Desde os primórdios, a enfermagem é exercida majoritariamente por mulheres, considerando-se que cuidados aos enfermos mostram-se cultural e socialmente apropriados para o trabalho feminino ${ }^{(20)}$.

Existem diferenças para o exercício profissional entre homens e mulheres, além de ser comum a sobrecarga de trabalho das mulheres, visto que, muitas vezes, conciliam atividades domésticas, às vezes em mais de um local de exercício profissional, o que representa desgaste físico e mental que, cedo ou tarde, pode repercutir em agravos à saúde ${ }^{(4,10,13-14,21-22)}$.

Dos 333 funcionários envolvidos em 662 episódios de afastamentos, o auxiliar de enfermagem foi a principal categoria de enfermagem acometida (82,3\%) em 1999 , resultado que se aproxima dos encontrados em outros estudos, nessa categoria ${ }^{(6,8,23)}$.

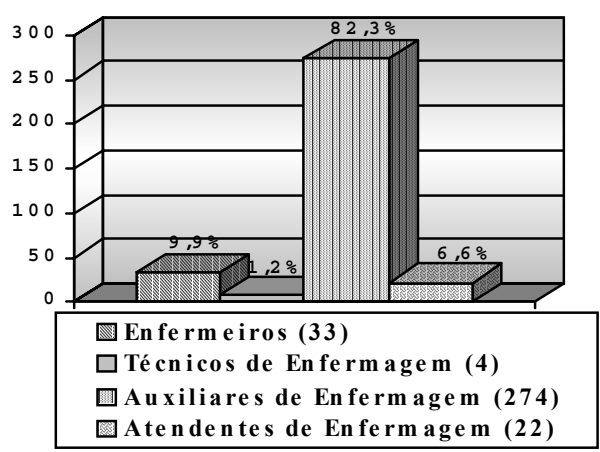

Figura 2 - Trabalhadores de enfermagem afastados do trabalho em 1999, segundo a categoria profissional 
Quanto à faixa etária, Tabela 1, predominaram os afastamentos entre trabalhadores com 21 a 40 anos: 261 (78,3\%), corroborando com os resultados encontrados em outras pesquisas $^{(6,10,13)}$. Esse mesmo resultado difere do obtido em estudo sobre doenças do trabalho e o exercício da enfermagem, onde ficou constatada maior freqüência de afastamentos do trabalho na faixa etária de 50 a 55 $\operatorname{anos}(86,4 \%)^{(3)}$.

Tabela 1 - Distribuição dos afastamentos por categoria profissional, segundo faixa etária e sexo, em um hospital geral de São José do Rio Preto, no ano de 1999

\begin{tabular}{|c|c|c|c|c|c|c|c|c|c|c|}
\hline \multirow{3}{*}{$\begin{array}{l}\text { FAIXA } \\
\text { ETÁRIA } \\
\text { (anos) }\end{array}$} & \multicolumn{10}{|c|}{ CATEGORIA PROFISSIONAL/SEXO } \\
\hline & \multicolumn{2}{|c|}{ Enfermeiro } & \multicolumn{2}{|c|}{$\begin{array}{l}\text { Técnico de } \\
\text { Enfermagem }\end{array}$} & \multicolumn{2}{|c|}{$\begin{array}{l}\text { Auxiliar de } \\
\text { Enfermagem }\end{array}$} & \multicolumn{2}{|c|}{$\begin{array}{l}\text { Atendente de } \\
\text { Enfermagem }\end{array}$} & \multicolumn{2}{|c|}{ TOTAL } \\
\hline & M & $\mathrm{F}$ & M & $\mathrm{F}$ & $\mathrm{M}$ & $\mathrm{F}$ & $\mathrm{M}$ & $\mathrm{F}$ & $\mathrm{N}$ & $\%$ \\
\hline 21 a 30 & - & 12 & 1 & 1 & 31 & 61 & 1 & 5 & 106 & 33,6 \\
\hline 31 a 40 & 1 & 19 & - & 1 & 22 & 100 & 1 & 5 & 150 & 44,7 \\
\hline 41 a 50 & - & 1 & - & 1 & $\overline{2}$ & 52 & 1 & 3 & 57 & 18,1 \\
\hline 51 a 60 & - & - & - & - & 1 & 4 & - & 6 & 18 & 3,3 \\
\hline 61 a mais & - & - & - & - & 1 & - & - & - & 1 & 0,3 \\
\hline TOTAL & 1 & 32 & 1 & 3 & 57 & 217 & 3 & 19 & 333 & 100,0 \\
\hline
\end{tabular}

$\mathrm{M}=$ Masculino; $\mathrm{F}=$ Feminino

Como mencionado anteriormente, os 333 funcionários deste estudo envolveram-se em 662 episódios de afastamentos, sendo 66 entre os 33 enfermeiros, 7 entre os 4 técnicos de enfermagem, 550 entre os 274 auxiliares de enfermagem e 39 entre os 22 atendentes de enfermagem, como se verifica na Tabela 2. Pode-se constatar que, dos 333 funcionários, 197 (59,1\%) afastaram-se apenas $1 \mathrm{vez}$, sendo que, dos outros funcionários que se afastaram mais de uma vez neste ano, a maioria teve de 2 a 4 afastamentos. Destaca-se um enfermeiro que se afastou 17 vezes e um auxiliar de enfermagem que teve 22 episódios de afastamentos do trabalho.

Tabela 2 - Distribuição dos afastamentos por número de episódios, segundo a categoria profissional, em um hospital geral de São José do Rio Preto, no ano de 1999

\begin{tabular}{|c|c|c|c|c|c|c|}
\hline \multirow{3}{*}{$\begin{array}{l}\text { EPISÓDIOS } \\
\text { (número de } \\
\text { vezes) }\end{array}$} & \multicolumn{6}{|c|}{ CATEGORIA PROFISSIONAL } \\
\hline & \multirow[t]{2}{*}{ Enfermeiro } & \multirow{2}{*}{$\begin{array}{l}\text { Técnico de } \\
\text { Enfermagem }\end{array}$} & \multirow{2}{*}{$\begin{array}{l}\text { Auxiliar de } \\
\text { Enfermagem }\end{array}$} & \multirow{2}{*}{$\begin{array}{l}\text { Atendente de } \\
\text { Enfermagem }\end{array}$} & \multicolumn{2}{|c|}{ TOTAL } \\
\hline & & & & & $\mathrm{N}$ & $\%$ \\
\hline 1 & 22 & 2 & 159 & 14 & 197 & 59,1 \\
\hline 2 & 4 & 1 & 55 & 4 & 64 & 19,3 \\
\hline 3 & 4 & 1 & 25 & 2 & 32 & 9,6 \\
\hline 4 & 2 & - & 18 & - & 20 & 6,0 \\
\hline 5 & - & - & 6 & 1 & 7 & 2,1 \\
\hline 6 & - & - & - & 1 & 1 & 0,3 \\
\hline 7 & - & - & 4 & - & 4 & 1,2 \\
\hline 8 & - & - & 2 & - & 2 & 0,6 \\
\hline 9 & - & - & 2 & - & 2 & 0,6 \\
\hline 10 & - & - & 2 & - & 2 & 0,6 \\
\hline 17 & 1 & - & - & - & 1 & 0,3 \\
\hline 22 & - & - & 1 & - & 1 & 0,3 \\
\hline TOTAL & 33 & 4 & 274 & 22 & 333 & 100,0 \\
\hline
\end{tabular}

Para melhor caracterizar os afastamentos, os resultados serão daqui por diante apresentados segundo os 662 episódios ocorridos no ano de 1999. Na Tabela 3 , onde se aborda o setor de trabalho, verifica-se que 36 $(54,5 \%)$ dos enfermeiros e $4(57,1 \%)$ dos técnicos de enfermagem afastados, atuavam em setor de internação. Chamam a atenção, nessa Tabela, os seguintes fatos: $163(29,6 \%)$ dos auxiliares e $8(20,5 \%)$ dos atendentes de enfermagem atuavam em UTI; que $339(61,6 \%)$ dos auxiliares de enfermagem e $15(38,5 \%)$ dos atendentes de enfermagem afastados atuavam em setores especializados e UTI e que, entre os atendentes de enfermagem, $13(33,3 \%)$ atuavam em unidade de CentroCirúrgico (CC), evidenciando desrespeito à legislação profissional da enfermagem ${ }^{(24)}$, pois tais trabalhadores de enfermagem não estão habilitados para atuarem em tais setores.

Tabela 3 - Distribuição dos afastamentos segundo categoria profissional e setor de trabalho, em um hospital geral de São José do Rio, no ano de 1999

\begin{tabular}{|c|c|c|c|c|c|c|}
\hline \multirow{3}{*}{$\begin{array}{l}\text { SETOR DE } \\
\text { TRABALHO }\end{array}$} & \multicolumn{6}{|c|}{ CATEGORIAPROFSSIONAL } \\
\hline & \multirow[t]{2}{*}{ Enfermeiro } & \multirow{2}{*}{$\begin{array}{l}\text { Técniøo de } \\
\text { Enfermagem }\end{array}$} & \multirow{2}{*}{$\begin{array}{l}\text { Auxiliar de } \\
\text { Enfermagem }\end{array}$} & \multirow{2}{*}{$\begin{array}{l}\text { Atendente de } \\
\text { Enfermagem }\end{array}$} & \multicolumn{2}{|c|}{ TOTAL } \\
\hline & & & & & $\mathrm{N}$ & $\%$ \\
\hline Un. Intemação & 36 & 4 & 119 & 10 & 169 & 25,5 \\
\hline Un. Especializada & 17 & - & 176 & 7 & 200 & 30,2 \\
\hline Uा & 6 & 3 & 163 & 8 & 180 & 27,2 \\
\hline Centro Cinúrgico & 2 & - & 82 & 13 & 97 & 14,7 \\
\hline Ambulatóio & 5 & - & 10 & 1 & 16 & 2,4 \\
\hline TOTAL & 66 & 7 & 550 & 39 & 662 & 100,0 \\
\hline
\end{tabular}

Un = Unidade de; UTI = Unidade de Terapia Intensiva

Características dos afastamentos: Os 662 afastamentos deste estudo são apresentados nas Tabelas 4 e 5 e Figuras 3 e 4 quanto a tipos, período e agrupamento dos problemas de saúde, conforme a distribuição da Classificação Internacional de Doença-CID-10 ${ }^{(25)}$ e enfoque dos afastamentos entre trabalhadores, segundo o sexo.

Pela Tabela 4, nota-se que, dos 662 episódios de afastamentos do trabalho, 88,4\% foram ocasionados por agravos à saúde (licença por motivo de saúde), nas quatro categorias de trabalhadores de enfermagem, 57 (86,4\%) dos enfermeiros, 7 (100\%) dos técnicos de enfermagem, $490(89,1 \%)$ dos auxiliares de enfermagem e 31 (79,5\%) dos atendentes de enfermagem. Analisando-se esses dados em consonância com a Tabela 3, verifica-se que os afastamentos acometeram, principalmente, funcionários que atuam em áreas mais complexas, como Unidades Especializadas (30,2\%), UTI $(27,2 \%)$ e Centro-Cirúrgico $(14,7 \%)$, onde são alvos para um grande número de transtornos de ordem física, química e psicológica, 
aumentando os riscos de agravos à saúde e afastamentos $^{(3,6,10)}$.

Tabela 4 - Distribuição dos tipos de afastamentos segundo categoria profissional em um hospital geral de São José do Rio Preto, no ano de 1999

\begin{tabular}{|c|c|c|c|c|c|c|}
\hline \multirow{3}{*}{$\begin{array}{l}\text { TIPOSDE } \\
\text { AFASTAMENTOS }\end{array}$} & \multicolumn{6}{|c|}{ CATEGORIAPROFSSIONAL } \\
\hline & \multirow[t]{2}{*}{ Enfermeiro } & \multirow{2}{*}{$\begin{array}{l}\text { Técrico de } \\
\text { Enfermagem }\end{array}$} & \multirow{2}{*}{$\begin{array}{l}\text { Auxiliar de } \\
\text { Enfermagem }\end{array}$} & \multirow{2}{*}{$\begin{array}{l}\text { Atendente de } \\
\text { Enfermagem }\end{array}$} & \multicolumn{2}{|c|}{ TOTAL } \\
\hline & & & & & $\mathrm{N}$ & $\%$ \\
\hline Acidente de & 1 & - & 40 & 7 & 48 & 7,2 \\
\hline Trabalho & & & & & & \\
\hline Licença-Gestante & 8 & - & 20 & 1 & 29 & 4,4 \\
\hline Licença-Saúde* & 57 & 7 & 490 & 31 & 585 & 88,4 \\
\hline TOTAL & 66 & 7 & 550 & 39 & 662 & 100,0 \\
\hline
\end{tabular}

* Licença por motivo de saúde

Quanto à duração dos afastamentos do trabalho, verifica-se, na Tabela 5, que, em 560 (84,6\%) dos episódios, o período foi de 1 a 10 dias, resultado que se aproxima do encontrado em outras pesquisas, embora se refiram a um período de 1 a 14 dias $(64,4 \%)^{(6,8)}$ e de até 15 dias $(74,2 \%)^{(3)}$. Quanto à preponderância dos afastamentos no período de 1 a 4 dias, que ocorreram em 427 (64,5\%) dos episódios neste estudo, encontrou-se, em outra pesquisa ${ }^{(26)}$, que $80 \%$ dos afastamentos tiveram duração de até três dias.

Tabela 5 - Distribuição dos afastamentos por categoria profissional, segundo o período de afastamento, em um hospital geral de São José do Rio Preto, no ano de 1999

\begin{tabular}{|c|c|c|c|c|c|c|}
\hline \multirow{3}{*}{ TEMPO } & \multicolumn{6}{|c|}{ CATEGORIAPROFSSIONAL } \\
\hline & \multirow[t]{2}{*}{ Enfermeiro } & \multirow{2}{*}{$\begin{array}{l}\text { Técnico de } \\
\text { Enfermagem }\end{array}$} & \multirow{2}{*}{$\begin{array}{l}\text { Auxiliar de } \\
\text { Enfermagem }\end{array}$} & \multirow{2}{*}{$\begin{array}{l}\text { Atendente de } \\
\text { Enfermagem }\end{array}$} & \multicolumn{2}{|c|}{ TOTAL } \\
\hline & & & & & $\mathrm{N}$ & $\%$ \\
\hline 1 a4 dias & 37 & 6 & 363 & 21 & 427 & 64,5 \\
\hline 5 a 10 dias & 15 & 1 & 109 & 8 & 133 & 20,1 \\
\hline 11 a 15 dias & 6 & - & 57 & 8 & 71 & 10,7 \\
\hline 16 a 29 dias & - & - & 2 & - & 2 & 0,3 \\
\hline 1 a 5 meses & 8 & - & 19 & 2 & 29 & 4,4 \\
\hline TOTAL & 66 & 7 & 550 & 39 & 662 & 100,0 \\
\hline
\end{tabular}

Verifica-se, na Tabela 6, que 585 afastamentos foram por agravos à saúde (licença-saúde), sendo que os principais problemas estavam relacionados a problemas geniturinários: 110 (18,8\%), e doenças mal definidas: 108 (18,6\%), que incluíram $29(50,9 \%)$ dos enfermeiros, 5 $(71,4 \%)$ dos técnicos de enfermagem, $180(36,7 \%)$ dos auxiliares de enfermagem. Já entre os atendentes de enfermagem, os problemas de saúde foram ocasionados, principalmente, por doenças dos órgãos dos sentidos: 6 (19,3\%), infecciosas e parasitárias: $5(16,1 \%)$ e do sistema respiratório: $5(16,1 \%)$.
Tabela 6 - Distribuição das licenças-saúde, segundo CID10 e categoria profissional, em um hospital geral de São José do Rio Preto, no ano de 1999

\section{CATEGORIA PROFISSIONAL}

\begin{tabular}{lcccccc} 
CID & Enfermeiro & $\begin{array}{c}\text { Técnico de } \\
\text { Enfermagem }\end{array}$ & $\begin{array}{c}\text { Auxiliar de } \\
\text { Enfermagem }\end{array}$ & $\begin{array}{c}\text { Atendente de } \\
\text { Enfermagem }\end{array}$ & \multicolumn{2}{c}{ TOTAL } \\
$\%$
\end{tabular}

DIP = Doenças Infecciosas e Parasitárias; $D N M B=$ Doenças Neoplasias Malignas e Benignas; DENM = Doenças Endócrinas, Nutricionais e Metabólicas; DSNTM = Doenças do Sistema Neurológico e Transtornos Mentais; DSOS = Doenças do Sistema dos Órgãos dos Sentidos; DSCV = Doenças do Sistema Cardiovascular; DSR = Doenças do Sistema Respiratório; DSD = Doenças do Sistema Digestivo; DST = Doenças do Sistema Tegumentar; DSO = Doenças do Sistema Osteomuscular; DSGUR = Doenças do Sistema Geniturinário e Reprodutor; DMD = Doenças Mal Definidas

Por fim, apresentam-se, nas Figuras 3 e 4, as características dos episódios de afastamentos entre os sujeitos deste estudo, considerando-se o sexo dos trabalhadores. Observa-se que $499(75,4 \%)$ dos trabalhadores, independente do sexo, afastaram-se só por agravos à saúde, correspondendo a $408(75,1 \%)$ entre as mulheres e 91 (76,5\%) entre os homens. Ressalta-se a ocorrência de 29 (5,3\%) licenças por gestação, e 34 (6,3\%) acidentes de trabalho. Dados da literatura revelam que a mulher não é mais suscetível a agravos da saúde nas ações profissionais, mas, sim, possui alterações fisiológicas e morfológicas que, geralmente, não são consideradas no planejamento do seu trabalho ${ }^{(27)}$.

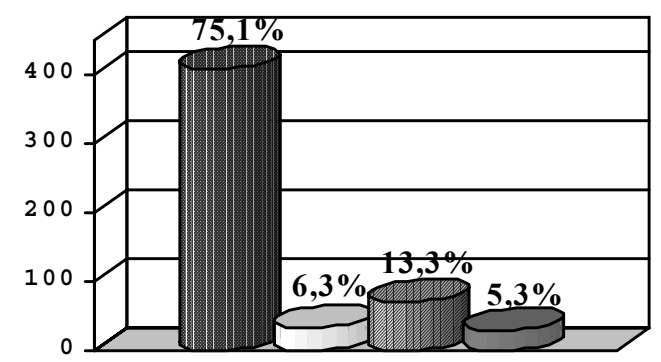
Só agravos à saúde (408)
$\square$ Acidentes de trabalho (34)
圆 Agravos à saúde + acidente trabalho (72)
$\square$ Licenças Gestantes (29)

Figura 3 - Tipos de afastamentos entre as mulheres $(n=543)$ 


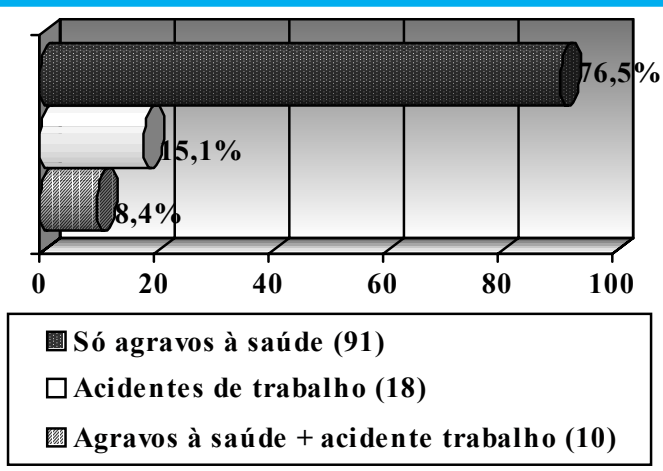

Figura 4 - Tipos de afastamentos entre os homens $(n=119)$

\section{CONCLUSÃO}

Segundo o objetivo definido para este estudo, verificou-se que ocorreram 662 episódios de afastamentos do trabalho em 1999, envolvendo 333 trabalhadores de enfermagem, principalmente mulheres, na categoria de auxiliar de enfermagem, com idade entre 21 a 40 anos.

Considerando-se o local de trabalho, houve predominância de afastamentos entre os enfermeiros e técnicos de enfermagem que atuavam em unidade de internação, enquanto que, entre os auxiliares e atendentes de enfermagem, afastaram-se mais aqueles que trabalhavam em setores especializados e UTI.

\section{REFERÊNCIAS BIBLIOGRÁFICAS}

1. Ferreira $\mathrm{ABH}$. Novo dicionário da língua portuguesa. $2^{\mathrm{a}} \mathrm{ed}$. Rio de Janeiro: Nova Fronteira; 1986.

2. Mendes R. O impacto dos efeitos da ocupação sobre a saúde de trabalhadores. 1. Morbidade. Rev Saúde Pública 1988; 22:311-26.

3. Bittencourt CM. Doenças do trabalho e o exercício da enfermagem. [dissertação]. Salvador (BA): Escola de Enfermagem/UFBa; 1993.

4. Alves DB. Causas de absenteísmo na enfermagem: uma dimensão do sofrimento no trabalho. [tese]. São Paulo (SP): Escola de Enfermagem/USP; 1996.

5. Benatti MCC. Acidentes do trabalho em um hospital universitário: um estudo sobre a ocorrência e os fatores de risco entre trabalhadores de enfermagem. [tese]. São Paulo (SP): Escola de Enfermagem/USP; 1997.

6. Silva DMPP. O adoecer dos trabalhadores de enfermagem: estudo dos problemas de saúde responsáveis pelo absenteísmo-doença em um hospital universitário. [dissertação]. Ribeirão Preto (SP): Escola de Enfermagem/ USP; 1999.

7. Vieira DFVB. Qualidade de vida no trabalho dos enfermeiros em um hospital de ensino. [dissertação]. Porto Alegre (RS): Faculdade de Ciências Econômicas/UFRS; 1993. 8. Silva DMPP, Marziale MHP. Absenteísmo de trabalhadores de enfermagem em um hospital universitário. Rev Latino-am Enfermagem 2000 out; 8(5):44-51.

9. Marziale MHP. Estudo da fadiga mental de enfermeiras atuantes em instituição hospitalar com esquema de trabalho em turnos alternados. [dissertação]. Ribeirão Preto (SP): Faculdade de Filosofia Ciências e Letras/USP; 1990.
A maioria dos episódios de afastamentos foram por agravos à saúde, $75,1 \%$ entre as mulheres e $76,5 \%$ entre os homens. As licenças-saúde ocorreram, principalmente, por problemas geniturinários e doenças mal definidas, entre os enfermeiros, técnicos e auxiliares de enfermagem, enquanto, entre os atendentes de enfermagem, os afastamentos relacionaram-se, principalmente, a problemas dos órgãos dos sentidos, doenças infecciosas e parasitárias e do sistema respiratório.

Os dados obtidos neste estudo permitem uma análise mais acurada da situação dos afastamentos do trabalho entre a equipe de enfermagem do hospital estudado, em 1999. Evidenciou-se que os funcionários afastados nas categorias de auxiliares e atendentes de enfermagem atuavam em locais para os quais não tinham preparo e qualificação legal, o que exige uma melhor análise da Chefia de Enfermagem quanto à situação de trabalho da equipe de enfermagem. Ainda, os resultados dão subsídios à Chefia de Enfermagem e à Administração Geral do Hospital para intervir e melhorar as condições de trabalho existentes para a equipe de enfermagem e, assim, diminuir os custos econômicos e sociais nesse contexto.

10. Pitta AMF. Hospital: dor e morte como ofício. São Paulo (SP): Hucitec; 1990.

11. Estryn-Behar M. Ergonomia y salud en el trabajo (I): el caso de las profissionais hospitaleiras. Rev ROL de Enfermaria 1996 jul/ago; (215-6):25-30.

12. Lopes GT, Spíndola T, Martins ERC. O adoecer em enfermagem segundo seus profissionais: estudo preliminares. Rev Enfermagem UERJ 1996 maio; 4(1):9-18. 13. Bulhões I. Riscos do trabalho de enfermagem. Rio de Janeiro (RJ): Correio Carioca; 1998.

14. Suazo SVV. Contribuição ao estudo sobre acidentes de trabalho que acometem as trabalhadoras de enfermagem em hospitais chilenos. [tese]. Ribeirão Preto (SP): Escola de Enfermagem/USP; 1999.

15. Aguir VE. Dolores de espalda. Rev ROL de Enfermeria $1995 \mathrm{abr} ;(200): 25-9$.

16. Busse M, Bridger B. Cost benefits of ergonomic intervention in a hospital: a preliminary study using Oxenburgh's productivity model. Curations 1997 Sep; 20(3):54-8.

17. Haag GS, Schuck JS, Lopes MJM. A enfermagem e a saúde dos trabalhadores. Goiânia (GO): AB; 1997.

18. Ortiz GCM, Patiño NAM. El estrés y su relacion com las condiciones de trabajo del personal de enfermaría. Invest Educ Enferm 1991 Sep; 2(9):83-99.

19. Almeida N Filho, Rouquayrol MZ. Fundamentos metodológicos da epidemiologia. In: Rouquayrol MZ. Epidemiologia e Saúde. $4^{a}$ ed. Rio de Janeiro (RJ): MEDSI; 1994. p.157-83.

20. Colliére MF. Promover a vida. Lisboa: Sindicato dos Enfermeiros Portugueses; 1989. 
21. Otero JJG. Riesgos del trabajo del personal sanitario. $2^{\mathrm{a}}$ ed. Madrid: McGraw-Hill; 1993.

22. Oguisso T. A mulher na força de trabalho: o trabalho da mulher enfermeira. Rev Enfermagem UERJ 1998 jun; 6(1):309-16.

23. Robazzi MLCC. Serviço de enfermagem: um estudo sobre os absenteísmos. Rev Bras Saúde Ocup 1990 jan/mar; 18(69):65-70.

24. Conselho Federal de Enfermagem (BR). O exercício da enfermagem nas instituições de saúde do Brasil: 1982/1983. Rio de Janeiro (RJ): COREn/ABEn; 1985.

25. Centro Colaborador da OMS (BR). Classificação Internacional de Doenças (CID-10). 5 $5^{\text {a }}$ ed. São Paulo (SP): Edusp; 1997.

26. Meira JBB. Absenteísmo por enfermidade: sugestões para o seu controle. Rev Bras Saúde Ocup 1982; 10(40):68-76.

27. Nogueira DP. Absentísmo-doença: aspectos epidemiológicos. [tese]. São Paulo (SP): Faculdade de Saúde Pública/USP; 1980. 